\title{
Off-axis holography and micro-optics improve lab-on-a-chip imaging
}

\author{
Zachary S Ballard, Yibo Zhang and Aydogan Ozcan
}

Light: Science \& Applications (2017) 6, e17105; doi:10.1038/lsa.2017.105; published online 22 September 2017

A pocket holographic slide enables the high-throughput screening of fluid samples by embedding micro-optics directly on a microchannel to achieve off-axis interferometric imaging. This holographic slide can be used to turn a conventional microscope into a phase-contrast imager or as a stand-alone compact imaging device by incorporating polymer lenses that are printed directly on-chip.

For decades, researchers have been developing lab-on-a-chip (LoC) technologies integrated with optics in an attempt to bring sensitive diagnostic measurements to practical and fully contained systems, which can be used at the point-of-care and in resource-limited settings ${ }^{1}$. Digital holography $(\mathrm{DH})$ is an imaging technique that is particularly well suited for LoC technologies because (i) it is label-free, which means that it does not require any sample pretreatment or labeling steps, thus forgoing the need for costly reagents and their refrigeration, and (ii) it can be easily incorporated with fluids to quickly screen large volumes of biofluids such as blood, serum, urine and saliva.

DH utilizes coherent or partially coherent illumination to generate interference between a reference wave and a wave scattered from an object. These holographic interference patterns can be recorded on a camera and then digitally refocused by using back-propagation algorithms to recover an in-focus image of the sample. Quantitative phasecontrast information of the sample can also be retrieved, thus enabling the high-resolution imaging of weakly scattering objects, such as microorganisms or pathogens, for example, in water samples. Various successful implementations of DH microscopy have been demonstrated in the literature. These compact and low-cost systems have been shown to be capable of imaging blood cells, waterborne parasites and even fast-moving self-propelled microorganisms, including sperm ${ }^{2-5}$.

However, as LoC technologies improve, engineers must grapple with an inherent trade-off between the two dominant DH modalities. $\mathrm{DH}$ with an in-line configuration offers high spatial resolution with an incredibly simple, low-cost and compact hardware implementation ${ }^{3}$; however, phase retrieval algorithms are required to eliminate the twin image artifact and recover the missing phase information of the sample. These algorithms are often computationally intensive, which might place a practical limitation on the frame rate of, for example, an optofluidic LoC device under a rapid flow condition. The second modality, the off-axis configuration, overcomes this phase retrieval

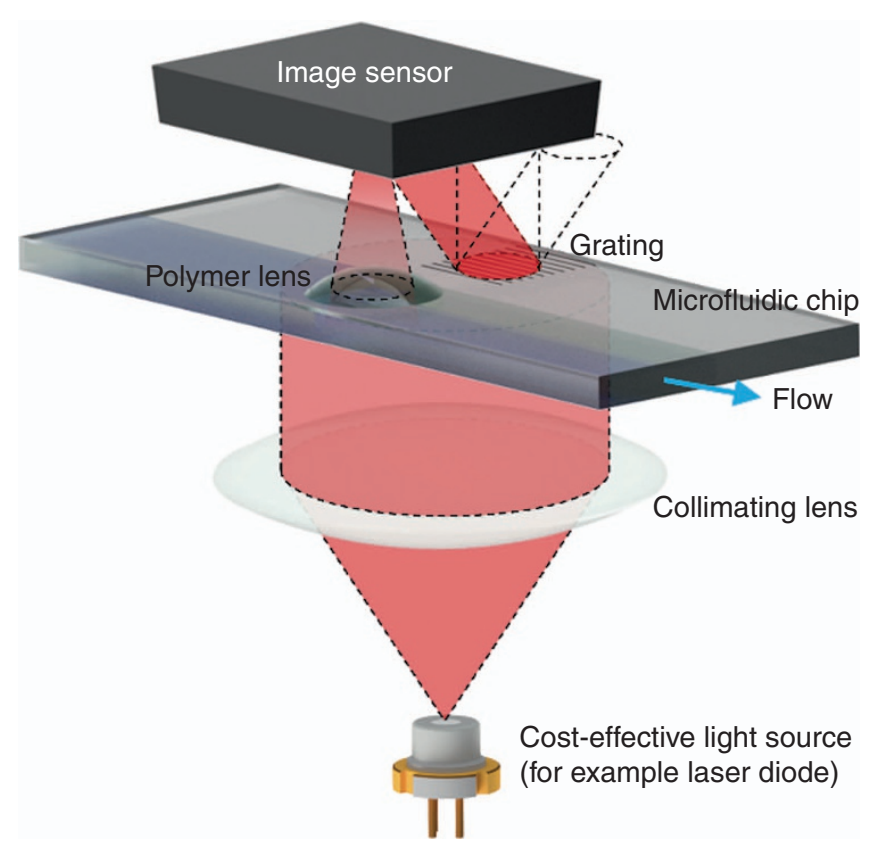

Figure 1 Off-axis holographic imager on a chip with laser diode illumination and micro-optics.

problem by utilizing a spatial carrier wave generated by a traditional interferometric set-up with spatially separated reference and sample arms. However, this benefit in terms of phase retrieval is compromised by a relative reduction in the effective space-bandwidth product of the reconstructed image and the need for relatively bulky and expensive optics that are sensitive to vibrations and misalignment ${ }^{6}$. Recently, the research group of Ferraro and co-workers demonstrated a LoC device that miniaturizes the interferometer optics to the chip level to achieve a robust and compact off-axis holographic imaging configuration (Figure 1), thus creating a 'pocket holographic microscope slide' that can single-handedly transform a conventional optical microscope into a phase-contrast imager? 
Their chip architecture implements a grating that is inscribed directly on a microfluidic channel to generate a diffraction beam, part of which acts as the off-axis arm of the interferometer. By using a single beam that impinges half on the grating and half on the channel alone, they demonstrate a self-aligned system that is tolerant to vibrations and misalignment. Furthermore, by using emerging fabrication techniques, such as imprint molding and pyroelectrohydrodynamic ink-jet printing, they were able to fabricate their 'pocket holographic slides' in a cost-effective and scalable manner ${ }^{7}$. Because both spatially separated arms of the interferometer can interact with the sample as it moves through the microfluidic channel, information from the object in either arm can be captured.

This LoC imaging technology smartly moves the interferometric optics on a chip in a robust self-aligned design, which can be replicated cost-effectively through scalable fabrication methods. The authors additionally demonstrated that by printing polymer lenses directly onto the chip, a highly compact and stand-alone holographic imaging device can be achieved (Figure 1). However, one hurdle remains: the Helium-Neon gas laser employed in this work must eventually be replaced by low-cost coherent sources such as laser diodes. The added challenges of a shorter coherence length, relatively weaker source stability and possible artifacts from chromatic dispersion need to be overcome to fully utilize the benefits of this LoC imaging device. To this end, future work exploring the use of laser diodes or LEDs will lead to a transformative imaging device for the cost-effective and highthroughput screening of fluid samples, further increasing diagnostic capabilities in resource-limited settings, among other applications.

\section{CONFLICT OF INTEREST}

The authors declare no conflict of interest.

1 Zhu HY, Isikman SO, Mudanyali O, Greenbaum A, Ozcan A. Optical imaging techniques for point-of-care diagnostics. Lab Chip 2013; 13: 51-67.

2 Kim MK. Digital Holographic Microscopy: Principles, Techniques, and Applications. New York: Springer, 2011.

3 Mudanyali O, Tseng D, Oh C, Isikman SO, Sencan I et al. Compact, light-weight and cost-effective microscope based on lensless incoherent holography for telemedicine applications. Lab Chip 2010; 10: 1417-1428.

4 Su TW, Xue L, Ozcan A. High-throughput lensfree 3D tracking of human sperms reveals rare statistics of helical trajectories. Proc Natl Acad Sci USA 2012; 109: 16018-16022.

$5 \mathrm{Xu} \mathrm{W}$, Jericho MH, Meinertzhagen IA, Kreuzer HJ. Digital in-line holography for biological applications. Proc Natl Acad Sci USA 2001; 98: 11301-11305.

6 Cuche E, Marquet P, Depeursinge C. Spatial filtering for zero-order and twin-image elimination in digital off-axis holography. Appl Opt 2000; 39: 4070-4075.

7 Bianco V, Mandracchia B, Marchesano V, Pagliarulo V, Olivieri F et al. Endowing a plain fluidic chip with micro-optics: a holographic microscope slide. Light Sci App/ 2017; 6: e17055.

(2) $(\$$ This work is licensed under a Creative Commons AttributionNonCommercial-NoDerivs 4.0 International License. The images or other third party material in this article are included in the article's Creative Commons license, unless indicated otherwise in the credit line; if the material is not included under the Creative Commons license, users will need to obtain permission from the license holder to reproduce the material. To view a copy of this license, visit http:// creativecommons.org/licenses/by-nc-nd/4.0/

(C) The Author(s) 2017 